\title{
REPULSIVE GRAVITY OF LIGHT BEAMS
}

\author{
GIOVANNI SPARANO AND GAETANO VILASI
}

Communicated by Abraham A. Ungar

\begin{abstract}
The analysis of some exact solutions of Einstein equations, describing gravitational waves produced by light, shows that there can be repulsion between light beams. This is due only to the spin- 1 character of the solutions and not to the introduction of other inputs external to General Relativity. Cosmological relativistic jets give an example of a such phenomenon.
\end{abstract}

\section{Introduction}

In this paper we study the possibility of a repulsive behavior in gravitational interaction, in particular between two light beams, due to the spin properties of some exact solutions of Einstein equations. More precisely we observe that there are exact solutions with spin- 1 which describe gravitational waves produced by light sources and give rise to a repulsive behavior. The arguments which actually attribute spin-2 to gravitational waves, relies on the assumption that the solutions be Fourier expandable and, as a consequence have no spin-1 components. However there exist $[7,23,31,32]$ (see Sections 2 and 3 ) physically meaningful solutions of Einstein equations which although not Fourier expandable are nonetheless finite energy solutions. An application of this result (see Section 4) is concerned with the numerical data related to cosmological relativistic jets.

Section 2 describes a family of exact solutions of Einstein equations, representing gravitational waves generated by a light beam or, more generally, by massless particles, and their physical properties.

Section 3 containes some historical remarks and with the Tolman, Ehrenfest, Podolsky [33] and Wheeler [36] results on gravitational repulsive behavior.

Section 4 deals with cosmological relativistic jets. 


\section{The Gravitational Interaction of Light}

\subsection{Geometric Properties}

In previous papers [7-10,27-29,34,35] a family of exact solutions $g$ of Einstein field equations, representing the gravitational wave generated by a beam of light, has been explicitly written

$$
g=2 f\left(\mathrm{~d} x^{2}+\mathrm{d} y^{2}\right)+\mu\left[(w(x, y)-2 q) \mathrm{d} p^{2}+2 \mathrm{~d} p \mathrm{~d} q\right]
$$

where $\mu(x, y)=A \Phi(x, y)+B$ (with $\Phi(x, y)$ a harmonic function and $A, B$ numerical constants), $f(x, y)=(\nabla \Phi)^{2} \sqrt{|\mu|} / \mu$, and $w(x, y)$ is a solution of the Euler-Darboux-Poisson equation

$$
\Delta w+\left(\partial_{x} \ln |\mu|\right) \partial_{x} w+\left(\partial_{y} \ln |\mu|\right) \partial_{y} w=\rho
$$

where $T_{\mu \nu}=\rho \delta_{\mu 3} \delta_{\nu 3}$ is representing the energy-momentum tensor and $\Delta$ is the Laplace operator in the $(x, y)$-plane.

Previous metrics are invariant for the non Abelian Lie agebra $\mathcal{G}_{2}$ of Killing fields

$$
X=\frac{\partial}{\partial p}, \quad Y=\mathrm{e}^{p} \frac{\partial}{\partial q}
$$

with

$$
[X, Y]=Y, \quad g(Y, Y)=0
$$

generating a two-dimensional distribution $\mathcal{D}$ whose orthogonal distribution $\mathcal{D}^{\perp}$ is integrable.

In the particular case $s=1, f=1 / 2$ and $\mu=1$, the above family is locally diffeomorphic to a subclass of Peres solutions and, by using the transformation

$$
p=\ln |u|, \quad q=u v
$$

can be written in the form

$$
g=\mathrm{d} x^{2}+\mathrm{d} y^{2}+2 \mathrm{~d} u \mathrm{~d} v+\frac{w}{u^{2}} \mathrm{~d} u^{2}
$$

with $\Delta w(x, y)=\rho$, and has the Lorentz invariant Kerr-Schild form

$$
g_{\mu \nu}=\eta_{\mu \nu}+V k_{\mu} k_{\nu}, \quad k_{\mu} k^{\mu}=0 .
$$




\subsection{Physical Properties}

\subsubsection{Wave Character}

The wave character and the polarization of these gravitational fields has been analyzed in many ways. For example, the Zel'manov criterion [37] was used to show that these are gravitational waves and the propagation direction was determined by using the Landau-Lifshitz pseudo-tensor. However, the algebraic Pirani criterion is easier to handle since it determines both the wave character of the solutions and the propagation direction at once. Moreover, it has been shown that, in the vacuum case, the two methods agree. To use this criterion, the Weyl scalars must be evaluated according to Petrov classification [24].

In the Newmann-Penrose formulation [22] of Petrov classification, we need a tetrad basis with two real null vector fields and two real spacelike (or two complex null) vector fields. Then, if the metric belongs to type $\mathbb{N}$ of the Petrov classification, it is a gravitational wave propagating along one of the two real null vector fields (Pirani criterion). Let us observe that $\partial_{x}$ and $\partial_{y}$ are spacelike real vector fields and $\partial_{v}$ is a null real vector but $\partial_{u}$ is not. With the transformation

$$
x \mapsto x, \quad y \mapsto y, \quad u \mapsto u, \quad v \mapsto v+\frac{w(x, y)}{2 u}
$$

whose Jacobian is equal to one, the metric (2) becomes

$$
g=\mathrm{d} x^{2}+\mathrm{d} y^{2}+2 \mathrm{~d} u \mathrm{~d} v+\mathrm{d} w(x, y) \mathrm{d} \ln |u| .
$$

Since $\partial_{x}$ and $\partial_{y}$ are spacelike real vector fields and $\partial_{u}$ and $\partial_{v}$ are null real vector fields, the above set of coordinates is the right one to apply for the Pirani's criterion. Since the only nonvanishing components of the Riemann tensor, corresponding to the metric (3), are

$$
R_{i u j u}=\frac{2}{u^{3}} \partial_{i j}^{2} w(x, y), \quad i, j=x, y
$$

these gravitational fields belong to Petrov type $\mathbb{N}$ [37]. Then, according to the Pirani's criterion, previous metric does indeed represent a gravitational wave propagating along the null vector field $\partial_{u}$.

It is well known that linearized gravitational waves can be characterized entirely in terms of the linearized and gauge invariant Weyl scalars. The non vanishing Weyl scalar of a typical spin-2 gravitational wave is $\Psi_{4}$. Metrics (3) also have as non vanishing Weyl scalar $\Psi_{4}$. 


\subsubsection{Spin}

Besides being an exact solution of the Einstein equations, the metric (3) is (for $w / u^{2} \ll 1$ ), also a solution of linearized Einstein equations, thus representing a perturbation of Minkowski metric $\eta=\mathrm{d} x^{2}+\mathrm{d} y^{2}+2 \mathrm{~d} u \mathrm{~d} v=\mathrm{d} x^{2}+\mathrm{d} y^{2}+\mathrm{d} z^{2}-\mathrm{d} t^{2}$ (where $u=(z-t) / \sqrt{2}, \quad v=(z+t) / \sqrt{2}$ ) with the perturbation, generated by a light beam or by a photon wave packet moving along the $z$-axis, given by

$$
h=\mathrm{d} w(x, y) \mathrm{d} \ln |z-t|
$$

whose non vanishing components are

$$
h_{0,1}=-h_{13}=-\frac{w_{x}}{(z-t)}, \quad h_{0,2}=-h_{23}=-\frac{w_{y}}{(z-t)} .
$$

\subsubsection{The Energy-Momentum Tensor}

A transparent method to determine the spin of a gravitational wave is to look at its physical degrees of freedom, i.e., the components which contribute to the energy [11]. One should use the Landau-Lifshitz (pseudo)-tensor $t_{\nu}^{\mu}$ which, in the asymptotically flat case, agrees with the Bondi flux at infinity [9]. It is worth to remark that the canonical and the Landau-Lifchitz energy-momentum pseudotensors are true tensors for Lorentz transformations. Thus, any Lorentz transformation will preserve the form of these tensors and this allows to perform the analysis according to the Dirac procedure. A globally square integrable solution $h_{\mu \nu}$ of the wave equation is a function of $r=k_{\mu} x^{\mu}$ with $k_{\mu} k^{\mu}=0$.

With the choice $k_{\mu}=(1,0,0,-1)$, we get for the energy density $t_{0}^{0}$ and the energy momentum $t_{0}^{3}$ the following result

$$
16 \pi t_{0}^{0}=\frac{1}{4}\left(u_{11}-u_{22}\right)^{2}+u_{12}^{2}, \quad t_{0}^{0}=t_{0}^{3}
$$

where $u_{\mu \nu} \equiv \mathrm{d} h_{\mu \nu} / \mathrm{d} r$. Thus, the physical components which contribute to the energy density are $h_{11}-h_{22}$ and $h_{12}$. Following the analysis of Dirac, we see that they are eigenvectors of the infinitesimal rotation generator $\mathcal{R}$, in the plane $x-y$, belonging to the eigenvalues $\pm 2 \mathrm{i}$. The components of $h_{\mu \nu}$ which contribute to the energy thus correspond to spin -2 .

In the case of the prototype of spin-1 gravitational waves (3), both Landau-Lifchitz energy-momentum pseudo-tensor and Bel-Robinson energy-momentum tensor [3, $4,26]$ single out the same wave components and we have

$$
\tau_{0}^{0} \sim c_{1}\left(h_{0 x, x}\right)^{2}+c_{2}\left(h_{0 y, x}\right)^{2}, \quad t_{0}^{0}=t_{0}^{3}
$$


where $c_{1}$ and $c_{2}$ are constants, so that the physical components of the metric are $h_{0 x}$ and $h_{0 y}$. Following the previous analysis one can see that these two components are eigenvectors of $\mathrm{i} \mathcal{R}$ belonging to the eigenvalues \pm 1 . In other words, metrics (3), which are not pure gauge since the Riemann tensor is not vanishing, represent spin-1 gravitational waves propagating along the $z$-axis at light velocity.

\subsubsection{Summarizing}

Globally square integrable spin-1 gravitational waves propagating on a flat background are always pure gauge and spin-1 gravitational waves which are not globally square integrable are not pure gauge.

It is always possible to write metric (3) in an apparently transverse gauge [31] but since these coordinates are no more harmonic this transformation is not compatible with the linearization procedure.

What truly distinguishes spin-1 from spin-2 gravitational waves is the fact that in the spin-1 case the Weyl scalar has a non trivial dependence on the transverse coordinates $(x, y)$ due to the presence of the harmonic function. This could led to observable effects on length scales larger than the characteristic length scale where the harmonic function changes significantly.

Indeed, the Weyl scalar enters in the geodesic deviation equation implying a non standard deformation of a ring of test particles breaking the invariance under of $\pi$ rotation around the propagation direction. Eventually, one can say that there should be distinguishable effects of spin-1 waves at suitably large length scales.

It is also worth to stress that the results of Aichelburg and Sexl, Felber and van Holten $[1,13,17]$ suggest that the sources of asymptotically flat $p p$-waves (which have been interpreted as spin-1 gravitational waves $[7,9]$ ) repel each other. Thus, in a field theoretical perspective, " $p p$ - gravitons" must have spin-1.

\subsection{Gravitoelectrodynamics}

Hereafter the spatial part of four-vectors will be denoted in bold and the standard symbols of three-dimensional vector calculus will be adopted.

Metric (3) can be written in the gravitoelectromagnetic form

$$
g=\left(2 \Phi^{(g)}-1\right) \mathrm{d} t^{2}-4\left(\mathbf{A}^{(g)} \cdot \mathrm{d} \mathbf{r}\right) \mathrm{d} t+\left(2 \Phi^{(g)}+1\right) \mathrm{d} \mathbf{r} \cdot \mathrm{d} \mathbf{r}
$$

where

$$
\mathbf{r}=(x, y, z), \quad 2 \Phi^{(g)}=h_{00}, \quad 2 A_{i}^{(g)}=-h_{0 i} .
$$




\subsubsection{Gravito-Lorentz Gauge}

In terms of $\Phi^{(g)}$ and $\mathbf{A}^{(g)}$ the harmonic gauge condition reads

$$
\frac{\partial \Phi^{(g)}}{\partial t}+\frac{1}{2} \nabla \cdot \mathbf{A}^{(g)}=0
$$

and, once the gravitoelectric and gravitomagnetic fields are defined in terms of g-potentials, as

$$
\mathbf{E}^{(\mathrm{g})}=-\nabla \Phi^{(g)}-\frac{1}{2} \frac{\partial \mathbf{A}^{(g)}}{\partial t}, \quad \mathbf{B}^{(\mathrm{g})}=\nabla \wedge \mathbf{A}^{(g)}
$$

one finds that the linearized Einstein equations resemble Maxwell equations. Consequently, being the dynamics fully encoded in Maxwell-like equations, this formalism describes the physical effects of the vector part of the gravitational field.

\subsubsection{Gravito-Faraday Tensor Field}

Gravitational waves can be also described in analogy with electromagnetic waves, the gravitoelectric and the gravitomagnetic components of the metric being

$$
E_{\mu}^{(g)}=F_{\mu 0}^{(g)}, \quad B^{(g) \mu}=-\varepsilon^{\mu 0 \alpha \beta} F_{\alpha \beta}^{(g)} / 2
$$

where

$$
F_{\mu \nu}^{(g)}=\partial_{\mu} A_{\nu}^{(g)}-\partial_{\nu} A_{\mu}^{(g)}, \quad A_{\mu}^{(g)}=-h_{0 \mu} / 2=\left(-\Phi^{(g)}, \mathbf{A}^{(g)}\right) .
$$

\section{Tolman-Erhenfest-Podolsky Problem}

\subsection{History}

The interest in repulsive gravity, or antigravity as it was usually called, goes back to the fifty's $[18,19,21]$.

The general point of view was that since gravitational interaction is mediated by a spin-2 particle, it can only be attractive and thus, to obtain a repulsive behavior, some other ingredient is required. The idea was then to explore the possibility of repulsive matter-antimatter gravity, but within the old quantum field theories there was no room for such a possibility.

The main arguments, reviewed in [21], were of various kinds including violation of energy conservation and disagreement with experiments of the Eötvös type due to the effects of antigravity on the vacuum polarization diagrams of atoms. 
More recently however, within the context of modern quantum field theories, it was proven that those arguments were no longer sufficient to exclude repulsive effects and the interest in antigravity increased again. For example, in [16] it was shown that in supergravity and string theory, due to dimensional reduction, the effective four-dimensional theory of gravity may show repulsive aspects because of the appearance of spin-1 graviphotons.

\subsection{Photon-Photon Scattering}

Photon-photon scattering can occur through the creation and annihilation of virtual electron-positron pairs and may even lead to collective photon phenomena. Photons also interact gravitationally but the gravitational scattering of light by light has been much less studied.

Purely general relativistic treatments of electromagnetic wave interactions have been made resulting in exact solutions $[14,15]$, but these calculations are different from pure scattering processes and do not address the interaction at single photon level.

It is not clear to what extent, calculations of the gravitational cross-section using QFT methods are consistent with classical GR. First studies go back to Tolman, Ehrenfest and Podolsky 1931 and, later, to Wheeler 1955 [33,36] who analysed the gravitational field of light beams and the corresponding geodesics in the linear approximation of Einstein equations. They discovered that null rays behave differently according to whether they propagate parallel or antiparallel to a steady, long, straight beam of light, but they did not provide a physical explanation of this fact.

Later, Barker, Bathia and Gupta [2], following a previous analysis of Barker, Gupta and Haracz [6], analyzed in QED the photon-photon interaction through the creation and annihilation of a virtual graviton in the center-mass system and they found that the interaction is eight times the "Newtonian" value plus a polarization dependent repulsive contact interaction and also obtained the gravitational cross sections for various photon polarization states.

Results of Tolman, Ehrenfest, Podolsky, Wheeler were clarified in part in [12], in the setting of classical pure General Relativity, using an approach based on a generalization to null rays of the gravitoelectromagnetic Lorentz force of linearized gravity.

They also extended the analysis to the realm of exact $p p$-wave solutions of the Einstein equations. Later, photon-photon scattering due to self-induced gravitational perturbations on a Minkowski background has been also analyzed by Brodin, Eriksson and Marklund [5] solving the Einstein-Maxwell system perturbatively to 
third order in the field amplitudes and confirming the dependence of differential gravitational cross section on the photon polarizations.

\subsection{Geodesic Motion}

The geodesic motion of a massive particle moving with four-velocity $v^{\mu}=(1, \underline{\mathrm{v}})$, $|\mathbf{v}| \ll 1$, in a light beam gravitational field characterized by gravitoelectric $\mathbf{E}^{(g)}$ and gravitomagnetic $\mathbf{B}^{(g)}$ fields, is determined (at first order in $|\mathbf{v}|$ ) by the acceleration

$$
\mathbf{a}^{(g)}=-\mathbf{E}^{(g)}-2 \mathbf{v} \wedge \mathbf{B}^{(g)} .
$$

The geodesic motion of a massless particle moving with velocity $v^{\mu}=(1, \mathbf{v})$, $|\mathbf{v}|=1$, in the light beam gravitational field, parallel(anti) to $z$-axis $\left(v_{j}= \pm \delta_{j 3}\right)$ is slightly different

$$
\mathbf{a}^{(g)}=-2\left(\mathbf{E}^{(g)}+\mathbf{v} \wedge \mathbf{B}^{(g)}\right) .
$$

There are two contributions, one by the light beam, which is the source of gravity, and the other by the test photon.

Since the gravitoelectric and gravitomagnetic fields corresponding to our metric are given by

$$
\mathbf{E}^{(g)}=\left(w_{x}, w_{y}, 0\right) / 4 u^{2}, \quad \mathbf{B}^{(g)}=\left(w_{y},-w_{x}, 0\right) / 4 u^{2}
$$

the 'gravitational acceleration' of a massless particle will be

$$
\mathbf{a}^{(g)}=-\left[w_{x}\left(1-v_{z}\right) \mathbf{i}+w_{y}\left(1-v_{z}\right) \mathbf{j}+\left(w_{x} v_{x}+w_{y} v_{y}\right) \mathbf{k}\right] / 2 u^{2} .
$$

The velocity $\mathbf{v}$ of a photon is determined by the null geodesics equations

$$
(h-1)-2 h v_{z}+(h+1) v_{z}^{2}=0
$$

which has two solutions

$$
v_{z}=1, \quad v_{z}=\frac{h-1}{h+1}=\frac{w-u^{2}}{w+u^{2}} .
$$

If the photon propagates parallel to the light beam, $v=(0,0,1)$, then

$$
\mathbf{a}^{(g)}=0
$$

and there is not attraction or repulsion (see also [38]).

If the photon does not propagate parallel to the light beam, the velocity will be $v=(h-1) /(h+1)$, then

$$
\mathbf{a}^{(g)}=-\nabla w / 2\left(w+u^{2}\right)
$$


and the force turns out to be attractive.

Thus, the lack of attraction found by Tolman, Ehrenfest, Podolsky comes out also from the analysis of the geodesical motion of a massless spin-1 test particle in the strong gravitational field of the light, neglecting however the gravitational field generated by that particle. An exhaustive answer could derive only determining the gravitational field generated by two photons, each one generating spin-1 gravitational waves. However, since helicity seems to play for photons the same role that charge plays for charged particles, two photons with the same helicity should repel one another. This repulsion turns out to be very weak and cannot be certainly observed in laboratory but it could play a relevant role at cosmic scale and could give not trivial contributions to the dark energy. Thus, together with gravitons (spin-2), one may postulate the existence of graviphotons (spin-1) and graviscalar (spin-0).

\section{Relativistic Jets}

Relativistic jets are extremely powerful jets of plasma emerging from presumed massive objects at the centers of some active radio galaxies and quasars. Their lengths can reach several thousand or even hundreds of thousands of light years. Among the different types of astrophysical jets, the most energetic ones are potential candidates to give rise to emission of gravitational waves. For example, highly relativistic jets should be associated with some sources of gamma ray bursts (GRBs) [25]. The impact of an ultra relativistic jet over the space-time metric can be studied starting from the extreme situation where the velocity of the particles in the beam is assumed to be equal to the velocity of light. The jet is then represented by a beam of null particles. For a flow of radiation of a null electromagnetic $(\mathrm{em})$ field along the $z$-axis, the (em) energy-momentum tensor macroscopic components $T_{\mu \nu}=F_{\mu \alpha} F_{\nu}^{\alpha}+\frac{1}{4} g_{\mu \nu} F_{\alpha \beta} F^{\alpha \beta}$ reduce to

$$
T_{00}=\frac{\rho}{z-c t}, \quad T_{03}=T_{30}=-\frac{\rho}{z-c t}, \quad T_{33}=\frac{\rho}{z-c t}
$$

where $\rho=\left(E^{2}+B^{2}\right) / 2$ represents the amplitude of the field, i.e., the density of radiant energy at point of interest. They are just the components in the coordinates $(t, x, y, z)$ of the energy-momentum tensor $T=\rho \mathrm{d} u^{2}$ of Section 2.

We assume then that the energy density is a constant $\rho_{0}$ within a certain radius $0 \leq r=\sqrt{x^{2}+y^{2}} \leq r_{0}$ and vanishes outside. Thus, the source represents a cylindrical beam with width $r_{0}$ and constitutes a simple generalization of a single null particle. 
Introducing back the standard coupling constant of Einstein tensor with matter energy-momentum tensor, we have

$$
\Delta w(x, y)=\frac{8 \pi G}{c^{4}} \rho .
$$

The cylindrical symmetry implies that $w(x, y)$ will depend only on the distance $r$ from the beam. A solution $w(r)$ of Poisson equation (7) satisfying the continuity condition at $r=r_{0}$ can be easily written as

$$
\begin{gathered}
w(r)=\frac{4 \pi G}{c^{4}} \rho_{0} r^{2}, \quad r \leq r_{0} \\
w(r)=\frac{8 \pi G}{c^{4}} \rho_{0} r_{0}^{2}\left[\ln \left(\frac{r}{r_{0}}\right)+\frac{1}{2}\right], \quad r>r_{0}
\end{gathered}
$$

or also

$$
w(r)=\frac{4 \pi G \rho_{0}}{c^{4}} r_{0}^{2} W(r)
$$

with $W(r)=r^{2} / r_{0}^{2}$ or $W(r)=1+\ln \left(\frac{r}{r_{0}}\right)^{2}$ depending on whether $r<r_{o}$ or $r>r_{0}$.

Thus, a photon moving antiparallel and external to the beam will experience at the space-time point $(t, x, y, z)$ a transversal gravitational attraction expressed by

$$
\mathbf{a}^{(g)}(t, x, y, z)=-\frac{16 \pi G}{c^{4}} \rho_{0} r_{0}^{2} \frac{\mathbf{r}}{r^{2}(z-c t)^{2}}
$$

where the speed of light $c$ has been reintroduced and the retardation is automatically accounted for. As a consequence of spin-1 of our wave and of QFT a photon moving parallel and external to the beam will experience at the space-time point $(t, x, y, z)$ a transversal gravitational repulsion given by

$$
\mathbf{a}^{(g)}(t, x, y, z)=\frac{16 \pi G}{c^{4}} \rho_{0} r_{0}^{2} \frac{\mathbf{r}}{r^{2}(z-c t)^{2}} .
$$

For jets which start with a small opening angle $\theta_{0} \leq 10^{-3}-10^{-4} \quad$ [25], it can be assumed that the width of the beam remains constant during the first stage of the jet expansion [20] and, for a beam-length $L=c \tau \approx 10^{6}-10^{7} \mathrm{Km}$ (a typical jet lasts $\tau \approx 10-100 \mathrm{~s}$ ), will be of the order of $r_{0}=L \theta_{0} \approx 10^{2}-10^{3} \mathrm{~km}$. The energy is of the order of $E=\approx 10^{44}-10^{45} \mathrm{~J}$, so that $\rho_{0}=E / L \approx 10^{37}-10^{39} \mathrm{~J} / \mathrm{km}$.

Replacing these values in equation (12) and taking $G / c^{4} \approx 10^{-44} \mathrm{~N}^{-1}$, we obtain for the transversal acceleration per unit length

$$
a^{(g)}(t, x, y, z)=\frac{10^{-5}}{r^{2}(z-c t)^{2}} \mathrm{~cm}^{-1}
$$


where $r=\sqrt{x^{2}+y^{2}}$ and $z$ are the distances, expressed in $\mathrm{cm}$, between the source and the point of interest and $t$ the observation time.

\section{Conclusions}

Repeating the above calculations for a laser beam in an interferometer of LIGO or VIRGO type, in the formula above we would get a factor of $10^{-50}$ instead of $10^{-5}$. Then, the repulsion (as well as the attraction) turns out to be very weak. However it could play a relevant role at cosmic scale and could give not trivial contributions to the dark energy.

At this point, together with gravitons (spin-2), one could postulate the existence of graviphotons (spin-1) and of graviscalar (spin-0) too. Through coupling to fermions, they might give forces depending on the barion number. These fields might give [30] two (or more) Yukawa type terms of different signs, corresponding to repulsive graviphoton exchange and attractive graviscalar exchange (range $\gg 200 m$ ). However, much more work must be done for a better understand of the role played by the gravitational field of the electromagnetic radiation and/or of null particles beams in the evolution of the universe.

\section{Acknowledgements}

We wish to thank the Italian Istituto Nazionale di Fisica Nucleare (INFN) and the Agenzia Spaziale Italiana (ASI) for partial support.

\section{References}

[1] Aichelburg A. and Sexl R., On the Gravitational Field of a Massless Particle, Gen. Rel. Grav. 2 (1971)303-312.

[2] Barker B., Bhatia M. and Gupta S., Gravitational Scattering of Light by Light, Phys. Rev. 158 (1967)1498-1499. Erratum: Barker B., Manjit S., Bhatia M., and Gupta S., Gravitational Scattering of Light by Light, Phys. Rev. 162 (1967) 1750-1750.

[3] Bel L., Sur la radiation gravitationnelle, C.R. Acad. Sci. (Paris) 247 (1958) 1094-1096.

[4] Bel L., Introduction d'un tenseur du quatrieme order, C.R. Acad. Sci. (Paris) 248 (1959) 1297-1300. 
[5] Brodin G., Eriksson D. and Maklund M., Graviton Mediated Photon-Photon Scattering in General Relativity, Phys. Rev. D 74 (2006) 124028-124032.

[6] Barker B., Gupta S. and Haracz R., One-Graviton Exchange Interaction of Elementary Particles, Phys. Rev. 149 (1966) 1027-1032.

[7] Canfora F., Vilasi G. and Vitale P., Nonlinear Gravitational Waves and Their Polarization, Phys. Lett. 545 (2002) 373-378.

[8] Canfora F. and Vilasi G., Spin-1 Gravitational Waves and Their Natural Sources, Phys. Lett. B 585 (2004) 193-199.

[9] Canfora F., Vilasi G. and Vitale P., Spin-1 Gravitational Waves, Int. J. Mod. Phys. B 18 (2004) 527-540.

[10] Canfora F., Parisi L. and Vilasi G., Nonlinear Gravitational Waves, Their Polarization and Realistic Sources, Theor. Math. Phys. 152 (2007) 1069-1080.

[11] Dirac PAM, General Theory of Relativity, Wiley, New York 1975.

[12] Faraoni V. and Dumse R., The Gravitational Interaction of Light: From Weak to Strong Fields, Gen. Rel. Grav. 31 (1999) 91-105.

[13] Felber F., Exact Antigravity-Field Solutions of Einstein's Equation, arXiv0803.2864; Felber F., Dipole Gravity Waves From Unbound Quadrupoles, arXiv1002.0351.

[14] Ferrari V. and Ibanez J., Gravitational Interaction of Massless Particles, Phys. Lett. A 141 (1989) 233-237.

[15] Ferrari V., Pendenza P. and Veneziano G., Beamlike Gravitational Waves and Their Geodesics, Gen. Rel. Grav. 20 (1988) 1185-1191.

[16] Fabbrichesi M. and Roland K., Strong Anti-Gravity, Nucl. Phys. B 388 (1992) 539-569.

[17] van Holten J., The Gravitational Field of a Light Wave, arXiv0808.0997.

[18] Morrison P. and Gold T., In: Essays on Gravity, Nine Winning Essays of the Annual Award $(1949$ - 1958) of the Gravity Research Foundation, Gravity Research Foundation, New Boston 1958, pp 45-50.

[19] Morrison P., Approximate Nature of Physical Symmetries, Am. J. Phys. 26 (1958) 358-368.

[20] de Rey Neto E. and de Araujo J., Aguiar O, A Gravitational Shock Wave Generated by a Beam of Null Matter in Quadratic Gravity, Class. Quant. Grav. 21 (2003) 1479-1488.

[21] Nieto M. and Goldman T., The Arguments Against 'Antigravity' and the Gravitational Acceleration of Antimatter, Phys. Rep. 205 (1991) 221-281 (Note: Errata issued in 1992 in volume 216).

[22] Penrose R., A Spinor Approach to General Relativity, Ann. Phys. 10 (1960) 171-201. 
[23] Peres A., Some Gravitational Waves, Phys. Rev. Lett. 3 (1959) 571-572.

[24] Petrov A., Einstein Spaces, Pergamon Press, New York 1969.

[25] Piran T., The Physics of Gamma-Ray Bursts, Rev. Mod. Phys. 76 (2004) 1143-2010; Sari R., Piran T. and Halpern J., The Physics of Gamma-Ray Bursts, Ap. J. 519 (1999) L17-L20; Piran T., The Physics of Gamma-Ray Bursts, Phys. Rep. 333 (2000) 529-553; Mészáros P., The Physics of GammaRay Bursts, Progress of Theoretical Physics Supplement, 136 (1999) 300320.

[26] Robinson I., On the Bel-Robinson Tensor, Class. Quantum Grav. 14 (1997) A331-A333.

[27] Sparano G., Vilasi G. and Vinogradov A., Gravitational Fields with a non Abelian Bidimensional Lie Algebra of Symmetries, Phys. Lett. B 513 (2001) 142-146.

[28] Sparano G., Vilasi G. and Vinogradov A., Vacuum Einstein Metrics with Bidimensional Killing Leaves I. Local Aspects, Diff. Geom. Appl. 16 (2002) 95120.

[29] Sparano G., Vilasi G. and Vinogradov A., Vacuum Einstein Metrics with Bidimensional Killing Leaves II. Global Aspects, Diff. Geom. Appl. 17 (2002) 15-35.

[30] Stacey F., Tuck G., and Moore G., Quantum Gravity: Observational Constraints on a Pair of Yukawa Terms, Phys. Rev. D 36 (1987) 2374-2380.

[31] Stephani H., General Relativity: An Introduction to the Theory of the Gravitational Field, Cambridge University Press, Cambridge 1996.

[32] Stephani H., Kramer D., MacCallum M., Honselaers C. and Herlt E., Exact Solutions of Einstein Field Equations, Cambridge University Press, Cambridge 2003.

[33] Tolman R., Ehrenfest P. and Podolsky B., On Gravitational Field Produced by Light, Phys. Rev. 37 (1931) 602-615.

[34] Vilasi G., Gravitational Waves as Exact Solutions of Einstein Field Equations, J. Phys. Conference Series 87 (2007) 012017-012029.

[35] Vilasi S, Sparano G. and Vilasi G., On the Gravity of Light, Class. Quantum Grav. 28 (2011) 195014-195025.

[36] Wheeler J., Geons, Phys. Rev. 97 (1955) 511-536.

[37] Zakharov V., Gravitational Waves in Einstein's Theory, Halsted Press, New York 1973.

[38] Zee A., Quantum Field Theory in a Nutshell ( $2^{\text {nd }}$ Edn), Princeton University Press, Princeton 2010. 
Giovanni Sparano

Dipartimento di Matematica

Università degli Studi di Salerno

Istituto Nazionale di Fisica Nucleare

Fisciano 84084, ITALY

E-mail address: Sparano@unisa.it

Gaetano Vilasi

Dipartimento di Fisica

Università degli Studi di Salerno

Istituto Nazionale di Fisica Nucleare

Fisciano 84084, ITALY

E-mail address: vilasiesa.infn.it 